\title{
The Advent of Digital Media Era and the Development Trend of National Animation Creation
}

\author{
Lina Zhang \\ Zaozhuang University, Zaozhuang, Shandong, 277160 \\ 290882312@qq.com
}

Keywords: Digital media era; Aesthetic approach; National animation

\begin{abstract}
In recent years, with the rapid development of digital technology and the widespread use of digital media, people's lifestyle and aesthetic trends have changed significantly. With the advent of the digital media era, Chinese national animation should be based on the active application of digital media technology, give full play to the aesthetic qualities of animation art, and create works of art with ethnic characteristics reflecting the characteristics of the times in order to meet people's aesthetic needs.
\end{abstract}

\section{Introduction}

With the rapid development of information technology, the application of digital media technology has become increasingly widespread. Digital media technology influences and changes people's life style and aesthetic tendency with its special artistic expression. Digital media technology does not only provide richer expressions for animation design and its creation, but also enables animations to express richer emotions flexibly and vividly, which brings a broad development space for national animation.

\section{Digital Media and Digital Media Era}

Compared with traditional media, digital media has increasingly shown its unique cultural value. In the eyes of some scholars, digital media is a medium that digitally generates, records, processes, disseminates, and retrieves information. ${ }^{[1]}$ Some scholars believe that information existing in the digital content as well as digital media which stores, transmits, receives information and their equipment can be called digital media. Digital media does not only include hardware facilities and related software, but also includes digital information content, etc. ${ }^{[2]}$ Currently, the definition of digital media, which is generally accepted by scholars, emphasizes the relationship between digital media and information technology. It holds that digital media cannot be separated into computer technology, network technology and mobile communication technology as a digital information carrier. At the same time, the content delivered by digital media technology is digitized information. From the history of the development of human society, the media is an important impetus to social development and has an important influence on the changes of people's daily contacts and on the changes of social lifestyles. The emergence and development of digital media is based on the rapid development of computer software and hardware, VLSI, high-capacity information storage, digital signal processing technology and Internet technology, and creates an important premise for a digital representation of text, which marks the arrival of digital media era.

In the age of digital media, digital languages have become the common language for communication among various media, which makes different media can be integrated on a unified digital platform. The exchange, integration and infiltration of information of various forms, sizes and flows have not only changed people's way of knowing the world, but also changed people's way of life and aesthetic approach.

\section{The Influence of Digital Media Times on People's Aesthetic Ways}

In the era of digital media, digital media is not only a tool or medium, but also has an important 
influence on people's aesthetic methods, making new aesthetic principles and new forms of art be possible.

In Terms of Artistic Appreciation, Encourage Active Participation of the Audience. In past artistic activities, artistic appreciation is actually the passive acceptance of artistic works by the audience. It is a one-way flow from creators, works of art to audiences, and the opinions of the audience cannot be effectively fed back to the creators in a timely manner. In the era of digital media, art appreciation has become a two-way interactive process. Art works do not only attract audiences to participate in appreciation activities, but also encourage communication and interaction between audiences and creators, and improve the artwork based on the opinions of the audience.

In the art appreciation activities in the era of digital media, the active participation of audience does not only change the traditional aesthetic distance theory, but also highlights the audience's important position in artistic activities. The audience cannot only communicate with the creators in a timely manner, but also can modify the artworks according to their preferences and appreciation habits to create new works that meet their aesthetic preferences. As a result, the boundary between the creator and the audience becomes blurred, and the audience begins to intervene and affect the art creation activities. For example, in the creation of an animation, the creator encourages the audience to participate in the creation of the work by telephone or text message, which has become an important way to increase the ratings of the animation work. The development of digital media technology has created more favorable conditions for audience participation. For example, Treehouse of Horror of the Simpsons' before Halloween of 2007 allowed the audience to edit their own Halloween artwork and communicate with other fans.

In the Emotional Experience, Emphasize the Unique Feelings of a Variety of Sensory Involvement. Because digital media is built on a combination of digital languages, 0 and 1, various media can communicate with one common platform and be replicated and distributed endlessly. Therefore, in the era of digital media, art creation can give full play to the advantages of multiple media to create unique works of art, thus bringing an unprecedented comprehensive experience to the audience.

For example, with the integrated use of multiple media, content in different media formats can be transplanted, collaged, and embedded at will. The artistic language is more plentiful, the content of works is more diverse, and the appreciation of works of art is significantly improved. For example, in the animation Happiness Interpretation Station, the unique animation language effectively compensates for the lack of visual performance of traditional folk arts. At the same time, humorous entertainment elements have been developed in the traditional folk arts, so that this animation produced a unique artistic aesthetic effect. With the further development of digital media technology, the trend of integrating various media into artistic creation will become more apparent, not only giving full play to the advantages of various media, but also giving the audience more and more rich and unique aesthetic experiences.

In Terms of Aesthetic Concepts, Pursuit the Integration of Art and Life. The extensive use of digital media technologies has not only enriched people's aesthetic experience, but also allowed arts to gradually infiltrate into everyday life. Art began to emerge from the noble status of being unattainable, returning to integration with everyday life. In this context, the aesthetic concept of art has shifted from the traditional pursuit of utilitarianism and spiritual experience to the satisfaction of physical pleasure and direct sensory pleasure. For example, in the domestic animation Boonie Bears, the confrontation between Briar and Bramble towards Logger Vick always ended with the failures of Logger Vick. The animated plot is simple and the narrative is plain, but it is widely acclaimed. One of the main reasons is that the artistic image and storyline created by creators which take emotional catharsis as the main goal are in line with the aesthetic concept of entertainment-oriented people who are currently pursuing pleasure experience. In the era of digital media, art often uses a lively and narrative style, and the content of daily life, with the help of exaggeration and distortion, creates a huge contrast with the traditional aesthetic concept of aesthetics and embodies aesthetic lifestyle tendency in the era of digital media. 


\section{The Main Strategies of National Animation Creation in Digital Media Era}

With the wide application of digital media technology and the advent of the digital media era, people's artistic concepts and lifestyles have undergone tremendous changes, and they have also provided new opportunities for the development of national animation. "With the development of network information technology, especially the development of the triple play of telephone networks, cable television networks and the Internet, high-speed, interactive and multimedia broadband networks will gradually become the mainstream media for culture, art and entertainment. This large confluence of cultures and technologies has brought a new era of animation art that shines in the light of the era of science and technology. It has opened a new stereoscopic, panoramic, interactive, immersive, and experiential future animation art. " ${ }^{[3]}$ Therefore, on the basis of digging deep into our national culture and traditional art resources, national animation should give full play to the advantages of digital media technology and strive to create artistic works with unique national character.

Enhance the Artistic Expression of National Animation. In the creation of animations, digital media technology is not only a production tool, but also promotes the revolution of animation creation ideas, making the animation style be more diversified. For example, due to the application of three-dimensional animation technology, the expression of the character's movement and even the texture of the skin produce an effect that is similar to that of a real person, which makes people produce a strong sense of reality and visual impact during the appreciation process; Anti-Treatment Technology makes animation works have a prominent sense of space, as well as the traditional hand-painted animation of the material, strokes and images and other characteristics. On the basis of extensive use of digital media technology, the animated art has a richer style. For example, due to the constraints of various factors, the traditional Chinese ink animation production techniques are gradually lost. With the aid of 3D rendering technology, creators can fully demonstrate the effects of ink and wash, providing an important opportunity for the inheritance of traditional ink animation styles.

At the same time, in the era of digital media, the traditional narrative methods have been unable to meet the requirements of the development of animation creation. Because in this era, there has been a "revolution in the way people feel." ${ }^{[4]}$ This "revolution" manifests itself in the subversion of traditional narrative and expression techniques. In the age of digital media, with the aid of certain technical means, animations, based on the extensive use of national traditional art forms, combined with the aesthetic concept of modern people, demonstrated the scenes and storylines of modern people's lives, and visualized the knowledge of national culture with Chinese characteristics. In order to improve the expressiveness of the national culture, diet, martial arts, folk custom, and other special nouns and genre styles appearing in traditional cultures are imprinted into the minds of the audience. For example, the character design of the animated film Nezha Conquers the Dragon King combines the creative skills of Shanxi murals and New Year paintings. It uses concise lines to outline the characters and express the change of the sea with the length, shortness, twists, and straightness of lines. These techniques are the creation techniques in the traditional painting arts of the nation, and the integration of each technique of digital and animation creates a character with distinctive national colors. ${ }^{[5]}$ The extensive application of digital media technology has made the animation art's expressive power be unprecedentedly enhanced, bringing audience a new visual experience.

Highlight the Uniqueness of the National Animation Art Form. Animation is a new type of art that emphasizes the integration of vision and hearing. In the animation, the sound is closely combined with images to create a unique artistic charm. As for animation, the factors such as sound and picture have nationality and uniqueness due to the influence of other mature artistic forms of the nation. For example, sound is one of the most direct carriers of animation art. Almost all characters in an animation need to rely on a specific form of sound to be fully represented. Therefore, in the animation creation, it is necessary to design a vivid and unique sound form to create a unique artistic effect. For example, the use of background music in the animated film Uproar In Heaven did not only fully demonstrate the unique value of the sound art form in the 
animation, but also highlighted the essence of the national opera. "The composer used the rhythm patterns of [26], [Kuaisanyan], [liushui], [daoban] and [yaoban], and the lyrical music showed changes which are scrambled in appearance but united in spirit. The use of Xiangyun which is the quintessence of China made the entire movie exude glory." ${ }^{, 6]}$ At the opening of the animation, drum and suona were played together to convey the joy of the monkeys. When Sun Wukong captured the treasure in Dragon Palace, the martial arts in traditional operas were used to create a heated atmosphere. The animation Three Monks also attached importance to the application of art forms. By cleverly playing the advantages of drums and cooperating with the monks' movements and expressions, it did not only realize the effective combination of images and sounds, but also highlighted the national characteristics of this animation.

In the era of digital media, the widespread use of various audio and video processing technologies does not only provide more extensive space for the design of audio and visual art forms, but also significantly enhances the narrative capabilities and expressiveness of sounds and other art forms in animation, which further highlights the uniqueness of the national animation art form.

Strengthen Humanistic Care of National Animation. Looking at contemporary animated films, whether they are American animations that pursue grand scenes, exotic stories, beautiful music, and digital special effects, or Japanese animations that focus on aesthetic images, vivid artistic images, and the impact of color vision, both of them are full of rich humanistic care and express deep thinking about life, the universe and the environment with rich philosophy. Excellent animation works do not only create a vivid image of art, tell the tortuous storyline, but also give the audience a strong spiritual shock, so that the audience's heart is purified and sublimated.

China's traditional aesthetics also pays attention to the spiritual world of human beings and values the unique value of art to life and society. This kind of humanistic care has always been associated with the requirements of the times and the needs of real life. Expressed in the creation of animations, it is to pay attention to people's actual living conditions and pay attention to ordinary people's daily life. Many animation works excavate "exotic" elements from the plain and unreal life, and use the artistic methods of fiction, distortion and exaggeration to inspire people's living wisdom and enrich people's spiritual life. For example, animations such as Uproar In Heaven, Nezha Conquers the Dragon King, Three Monks, and Little Catfish Jumping Dragon Gate have drawn people's familiar folk stories and used simple, easy-to-understand methods to arouse people's sympathy, conveysing a profound philosophy of life.

"Art is based on human emotions. Art aesthetics are based on what people need. Art is the creation of deep emotions by all means of human beings. It is a higher level interpretation of truth, goodness, and beauty in the human mind." ${ }^{[7]}$ In the era of digital media, the rapid development of computer technology and network technology have provided unlimited possibilities for nationsl animation. From the portrayal of characters' scenes to the rendering of grand scenes, to the portrayal of characters' expressions and behaviors, animations are not only more vivid and expressive, but also fully demonstrate unique humanistic connotations due to the use of digital media technology. The involvement of digital media technology does not only greatly expand the artistic imagination of animation, but also enriches animation production techniques, improves animation production and communication methods, and significantly enhances the artistic appeal of animation. Therefore, the wide application of digital media technology, based on enriching the expression form and artistic language of animation art, conveys deep reflections on nationality, culture, and life.

\section{Conclusion}

With the wide application of digital media technology, people's daily life and aesthetic styles have undergone tremendous changes. In the era of digital media, the issue of creating national animations has drawn great public concern. National animation should actively apply digital media technology to adapt to the development of people's aesthetic concept of art and strive to create animation works that reflect the characteristics of the digital media era and national characteristics. 


\section{References}

[1] Huifen Liu. Digital Media - Technology, Application, and Design [M]. Beijing: Tsinghua University Press, 2003: 2.

[2] Hong Wang. Digital Media Analysis [M]. Chongqing: Southwest China Normal University Press, 2006: 7.

[3] Xiangzhong Liao. Development Trend of Cartoon in the Digital Media Era [J]. Seeking, 2010, (14): 45-46.

[4] Sida Li. History of Digital Media Art [M]. Beijing: Tsinghua University Press, 2008: 241.

[5] Hongming Li. Study on the Living Inheritance of Folk Art from the Perspective of Intangible Cultural Heritage [J]. Cultural Disputes, 2014, (4): 184-187.

[6] Yi Zheng, Jintao Qian. Boosting and appreciating traditional Chinese Painting--Comments of Chinese Animation Music[J] Journal of Jilin Arts Institute, 2004, (4): 49-60.

[7] Qian Fu. Art Issues in Intelligent Survival [J]. Literature and Art Research, 2003, (1): 58-61.

About the author: Lina Zhang (1983 -), Female, Jining, Shandong; Master; Lecturer, Research direction: animation.

Address: Institute of Media, Zaozhuang College, No. 1, Beian Road, Shizhong District, Zaozhuang City, Shandong, 277160 